\title{
Bioactive components of Glycyrrhiza uralensis mediate drug functions and properties through regulation of CYP450 enzymes
}

\author{
HAO CHEN $^{1 *}$, XIAOMEI ZHANG $^{2 *}$, YIFAN FENG $^{2}$, WEN RUI $^{2}$, ZHONGFENG SHI $^{2}$ and LIRONG WU ${ }^{3}$ \\ ${ }^{1}$ Department of Pharmacy, Qilu Hospital of Shandong University, Jinan, Shandong 250012; ${ }^{2}$ Center Laboratory, Guangdong \\ Pharmaceutical University; ${ }^{3}$ Guangdong Provincial Key Laboratory of Pharmaceutical Bioactive Substances, \\ Guangdong Pharmaceutical University, Guangzhou, Guangdong 510006, P.R. China
}

Received June 24, 2013; Accepted April 14, 2014

DOI: $10.3892 / \mathrm{mmr} .2014 .2331$

\begin{abstract}
Glycyrrhiza uralensis (G. uralensis) is a common medicinal plant that has mainly been used to modulate the pharmaceutical activity of herbal medicines. Although G. uralensis has been shown to affect the expression and activity of the key metabolic enzyme cytochrome P450 (CYP450), the detailed mechanism of this process has yet to be elucidated. The present study aimed to elucidate the effects of bioactive components of G. uralensis on different isoforms of CYP450 and determine the ability of these components to modulate drug properties. In the present study, mRNA levels of CYP1A2, CYP2D6, CYP2E1, and $C Y P 3 A 4$ were investigated by quantitative polymerase chain reaction (qPCR) in HepG2 cells following treatment with the major bioactive compounds of G. uralensis. The activity of CYP450 enzymes was investigated in human liver microsomes using the cocktail probe drug method, and the metabolites of specific probes were detected by UPLC-MS/MS. The effects of $G$. uralensis on CYP450 were assessed using bioinformatics network analysis. Several compounds from G. uralensis had various effects on the expression and activity of multiple CYP450 isoforms. The majority of the compounds analysed the inhibited expression of CYP2D6 and CYP3A4. Several CYP isoforms were differentially modulated depending on the specific compound and dose tested. In conclusion, the present
\end{abstract}

Correspondence to: Dr Zhongfeng Shi, Center Laboratory, Guangdong Pharmaceutical University, 280 Waihuan East Road, High Education Mega Center, Guangzhou, Guangdong 510006, P.R. China

E-mail: shizhf@139.com

Dr Lirong $\mathrm{Wu}$, Guangdong Provincial Key Laboratory of Pharmaceutical Bioactive Substances, Guangdong Pharmaceutical University, 280 Waihuan East Road, High Education Mega Center, Guangzhou, Guangdong 510006, P.R. China

E-mail: 30872950@qq.com

${ }^{*}$ Contributed equally

Key words: cytochrome P450, Glyrrhiza uralensis, qPCR, cocktail assay, drug efficacy regulation study suggested that $G$. uralensis influenced the expression and activity of CYP450 enzymes. Therefore, caution should be taken when $G$. uralensis is co-administered with drugs that are known to be metabolized by CYP450. This study contributed to the knowledge of the mechanisms by which this medicinal plant, commonly known as licorice, modulates drug efficacy.

\section{Introduction}

Cytochrome P450 (CYP450) enzymes constitute a superfamily of membrane-bound heme proteins that catalyse the oxidative metabolism of a variety of endogenous compounds, including steroids, fatty acids, and prostaglandins (1), and exogenous compounds, including drugs, carcinogens, agrochemicals (2-4), and environmental pollutants (5-7). Seven human isoforms of CYP (CYP1A2, -2C9, -2C18, -2C19, -2D6, -2E1 and -3A4) metabolize $>90 \%$ of drugs currently used in the clinic (8). Alternatively, certain drugs are able to modify the expression and activity of CYP450 enzymes, thereby changing drug efficacy and pharmacokinetics. Various in vitro models have been used to assess CYP induction, including precision-cut liver slices $(9,10)$, primary hepatocytes $(11,12)$, and reporter gene constructs $(13,14)$. In particular, HepG2 cells retain the expression of all human CYPs (15) and are frequently used to evaluate the effects of xenobiotics on CYP450 expression. Additionally, the cocktail method is the most predominant technique used for studies on CYP450 conducted on human liver microsomes (HLMs).

Medicinal plants have been used to treat numerous types of diseases for thousands of years in China. In particular, the use of medicinal plants combined with conventional medicine is common in the treatment of chronic diseases, cancers, immunological disorders and infectious diseases. However, in contrast to the popular presumption that 'natural is safe', medicinal plants may have significant side effects. Additionally, there is an increasing concern for the risk of drug-drug interactions when medicinal plants are administered concomitantly with conventional medicines (16-19).

Glycyrrhiza uralensis (G. uralensis), also known as Chinese licorice, is a Traditional Chinese Medicine (TCM) that has been used in the clinic for centuries. Its functions include regulating drug properties, improving spleen function and blood circulation and reducing cough. The main bioactive components 
of $G$. uralensis are triterpene saponins and various types of flavonoids, including glycyrrhetinic acid (GA), glycyrrhizic acid (GL), liquiritigenin (L), isoliquiritigenin (IL), liquiritin (LG) and licochalcone A (LA). These components possess various biological activities, including antiulcer, anti-inflammatory, anti-allergic, antichrombotic, antidiabetic, hepatoprogenic and neuroprotective activities $(20,21)$.

Results of previous studies have shown that $G$. uralensis or its components activate the nuclear receptor PXR, inducing CYP3A and affecting lidocaine pharmacokinetics (22), and inhibit CYP2E1 expression when administered prior to carbon tetrachloride, exerting a protective effect on liver injury (23). However, the specific CYP450 isoforms involved and the effects of specific $G$. uralensis components have yet to be identified. Thus, G. uralensis has not currently been adopted for common use in clinical practice.

The present study aimed to investigate the effects of the main bioactive constituents of $G$. uralensis on the expression and activity of CYP isoforms.

\section{Materials and methods}

Chemicals. GA and LG were obtained from the National Institute for the Control of Pharmaceutical and Biological Products (Beijing, China). GL, L, IL, and LA were purchased from Shanghai Tauto Biotech (Shanghai, China). All the above constituents of G. uralensis were dissolved in DMSO (MP Biomedicals, Strasbourg, France). 3-(4,5-Dimethylthiazol-2-yl)-2,5-diphenyl tetrazolium bromide (MTT, Sigma-Aldrich Co., St. Louis, MO, USA) was dissolved in PBS (pH 7.4). Melatonin, dextromethorphan, coumarin and omeprazole are purchased from Wako (Osaka, Japan). Tolbutamide, chlozoxazone, phenacetin, amodiaquine, 6-hydroxymelatonin, and dextrophan were purchased from Sigma-Aldrich. Desmethylomeprazole, 6-hydroxychlozoxazone, 4-hydroxy tolbutamide, sulfone omeprazole, and 5-hydroxyomeprazole were purchased from International Laboratory (San Francisco, CA, USA).

Cell culture and cytotoxicity assay. Human hepatoma HepG2 cells were obtained from the Chinese Academy of Sciences Committee Type Culture Collection Cell Bank/CAS Shanghai Institute for Biologic Science Cell Resource Center (Shanghai, China) and maintained in Williams' Medium E supplemented with $10 \%$ fetal calf serum, $100 \mathrm{IU} / \mathrm{ml}$ penicillin $\mathrm{G}$, and $100 \mu \mathrm{g} / \mathrm{ml}$ streptomycin. The cells were maintained in $25-\mathrm{cm}^{2}$ flasks at $37^{\circ} \mathrm{C}$ in a humidified incubator with $5 \% \mathrm{CO}_{2}$.

The cytotoxic effects of the compounds on HepG2 cells were evaluated by measuring the metabolic activity using the MTT assay. Cells were grown in 96-well plates $\left(\sim 1 \times 10^{4}\right.$ cells/well) for $24 \mathrm{~h}$. The medium was replaced with fresh medium containing serial dilutions of the test compound $(0-100 \mu \mathrm{M})$. For all treatments, the final concentration of DMSO did not exceed $0.01 \%(\mathrm{v} / \mathrm{v})$. Following incubation for $24 \mathrm{~h}$, the medium was removed, $20 \mu 1 \mathrm{MTT}$ ( $5 \mathrm{mg} / \mathrm{ml}$ in PBS) was added to each well, and the cells were incubated for an additional $4 \mathrm{~h}$ at $37^{\circ} \mathrm{C}$ at $5 \% \mathrm{CO}_{2}$. The supernatant was then removed, and $150 \mu \mathrm{l}$ DMSO was added to each well. The absorbance at $490 \mathrm{~nm}$ was recorded using a BIO-TEK spectrophotometer.
RNA extraction, cDNA synthesis, and quantitative polymerase chain reaction $(q P C R)$. HepG 2 cells were seeded in 12-well plates and cultured until they reached $70-80 \%$ confluence. The medium was then replaced with fresh medium containing 25 or $50 \mu \mathrm{M}$ of different test compounds dissolved in DMSO. Following $24 \mathrm{~h}$ of incubation, total RNA was isolated using TRIzol according to the manufacturer's instructions (Takara Bio Inc., Otsu, Japan) and was quantified by measuring the absorbance at 260/280 nm. First-strand cDNA synthesis was performed using the High-Capacity cDNA Reverse Transcription kit (Applied Biosystems, USA) according to the manufacturer's instructions. Briefly, $2 \mu \mathrm{g}$ total RNA from each sample was added to a mixture of $2.0 \mu 1$ 10X RT buffer, $0.8 \mu 125 \mathrm{X}$ dNTP mix (100 mM), $1.0 \mu \mathrm{l}$ MultiScribe reverse transcriptase, $1.0 \mu \mathrm{l}$ Universal RT primer (20 $\mu \mathrm{M} 5$ '-aagc cgagacgacgacagacttttttttt tttttttttttVVN-3') and RNase-free water. The reaction mixture $\left(20 \mu \mathrm{l}\right.$ ) was incubated at $25^{\circ} \mathrm{C}$ for $10 \mathrm{~min}, 37^{\circ} \mathrm{C}$ for $120 \mathrm{~min}$, $85^{\circ} \mathrm{C}$ for $5 \mathrm{~min}$ and then cooled to $4^{\circ} \mathrm{C}$ for the final step. For all experiments, cDNA was diluted to a concentration equivalent to $20 \mathrm{ng} / \mathrm{ml}$ RNA. Synthesized cDNA was stored at $-20^{\circ} \mathrm{C}$.

qPCR was performed using the Bio-Rad IQ5 system (Bio-Rad, Hercules, CA, USA). PCR assays were performed in 96-well optical reaction plates. To avoid the impact of DNA contamination, primer pairs were designed to span an intron-exon boundary. Reaction mixtures $(25 \mu \mathrm{l})$ contained $0.1 \mu 1$ each (stock concentration, $10 \mu \mathrm{M}$ ) of the forward and reverse primers (final concentration of each primer, $40 \mathrm{nM}$; Table I), $12.5 \mu$ l Power SYBR-Green Universal Master Mix (Applied Biosystems, Beijing, China), $10.3 \mu \mathrm{l}$ nuclease-free water and $2 \mu \mathrm{l}$ cDNA solution. The reaction conditions were as follows: Initiation at $95^{\circ} \mathrm{C}$ for $10 \mathrm{~min}$, followed by 50 cycles of denaturation at $95^{\circ} \mathrm{C}$ for $15 \mathrm{sec}$ and annealing/extension at $60^{\circ} \mathrm{C}$ for $1 \mathrm{~min}$, followed by a dissociation step. Expression levels were normalized to the expression of the housekeeping gene, $G A P D H$, and relative expression was calculated using the $2^{-\Delta \Delta \mathrm{Ct}}$ method (24). Data are presented as the fold change relative to GAPDH expression.

HLM metabolic assay. HLMs were obtained from iPhase Pharmaceutical Services (Beijing, China). Microsomes were incubated with $500 \mu \mathrm{g} / \mathrm{ml}$ human microsomal protein in $0.1 \mathrm{M}$ potassium phosphate buffer ( $\mathrm{pH} 7.4), 1 \mathrm{mM}$ nicotine amide dinucleotide phosphate (NADPH), and substrates at the indicated concentrations. Substrates and their final concentration for incubation were as follows: Melatonin $(6 \mu \mathrm{M})$, coumarin $(4 \mu \mathrm{M})$, tolbutamide $(30 \mu \mathrm{M})$, omeprazole $(20 \mu \mathrm{M})$, dextromethorphan $(0.6 \mu \mathrm{M})$ and chlozoxazone $(30 \mu \mathrm{M})$. The first dilution of the substrate was produced in appropriate solvents, i.e., methanol (melatonin, coumarin, omeprazole), water (dextromethorphan), DMSO (tolbutamide) or $60 \mathrm{mM}$ potassium hydroxide solution (chlozoxazone), and subsequent dilutions were produced in $0.1 \mathrm{M}$ phosphate buffer ( $\mathrm{pH}$ 7.4). The final amount of solvent in the incubation mixture was $<0.05 \%(\mathrm{v} / \mathrm{v})$. The final concentration of each compound tested (i.e., GL, GA, L, IL, LG and LA), as well as the mixture containing all the compounds at the same ratio (GC), was $25 \mu \mathrm{M}$. The reaction mixture, at a final volume of $250 \mu \mathrm{l}$, was pre-incubated for $2 \mathrm{~min}$ at $37^{\circ} \mathrm{C}$ in a water bath prior to initiation of the reaction by the addition of NADPH. Following incubation for $20 \mathrm{~min}$, the reaction was terminated by the addition of $100 \mu \mathrm{l}$ acetonitrile containing $0.5 \mu \mathrm{M}$ 
Table I. Primers used for the analysis of gene expression by qPCR.

\begin{tabular}{llc}
\hline Gene & \multicolumn{1}{c}{ Primers } & Amplicon size (bp) \\
\hline GADPH & Forward: TCTCCCCTCCTCACAGTTGC & \\
& Reverse: AAGCCGAGACGACGACAGAC & 143 \\
FYP1A2 & Forward: AGCTTCTCCTGGCCTCTGC & \\
CYP2D6 & Forward: CGCATCCCTAAGGGAACGA & 88 \\
CYP2E1 & Reverse: TCCCAGACGGCCTCATCCT \\
CYP3A4 & Forward: GCAAGAGATGCCCTACATGGA & 68 \\
& Reverse: GGGCACGAGGGTGATGAA & 64 \\
\hline
\end{tabular}

qPCR, quantitative polymerase chain reaction; bp, base pairs; CYP, cytochrome P.

Table II. Substrates, CYP-specific model reactions and substrate-extracted ions in the cocktail.

\begin{tabular}{lcccc}
\hline Substrate & CYP & Reaction & Metabolite & Extracted ion (m/z) \\
\hline Melatonin & $1 \mathrm{~A} 2$ & 6-Hydroxylation & 6-OH-MEL & 249 \\
Coumarin & $2 \mathrm{~A} 6$ & 7-Hydroxylation & 7-OH-COU & 163 \\
Amodiaquine & $2 \mathrm{C} 8$ & Desethylation & deEt-AMO & 328 \\
Tolbutamide & $2 \mathrm{C} 9$ & Methylhydroxylation & OH-TOL & 287 \\
Omeprazole & $2 \mathrm{C} 19$ & 5-Hydroxylation & OH-OME & 362 \\
& $2 \mathrm{C} 19$ & Demethylation & Dem-OME & 332 \\
& $3 \mathrm{~A} 4$ & Sulfoxidation & SO-OME & 362 \\
\hline
\end{tabular}

CYP, cytochrome P; MEL, melatonin; COU, coumarin; AMO, amodiaquine; TOL, tolbutamide; OME, omeprazole.

phenacetin as an internal standard for each drug metabolite. The sample was then cooled in an ice bath to precipitate the protein. The mixture was vortexed and centrifuged at $14000 \mathrm{x} \mathrm{g}$ for $10 \mathrm{~min}$. The supernatant was analyzed using ultra performance liquid chromatography time of flight mass spectrometry (UPLC/TOF-MS).

Cocktail assay. For the cocktail assay, we used liquid chromatography-tandem mass spectrometry (LC-MS/MS) analysis. The analytical column was a Waters ACQUITY UPLC BEH C18 column $(1.7 \mu \mathrm{m}, 2.1 \times 5 \mathrm{~mm}$; Waters, Milford, MA, USA) with a C18 $1.7 \mu \mathrm{m}$ VanGuard 3/PK 2.1 x5 mm pre-column (Waters). The flow rate was $0.35 \mathrm{ml} / \mathrm{min}$, and the column temperature was $30^{\circ} \mathrm{C}$. The eluents used were (A) aqueous $1 \%$ formic acid and $10 \mathrm{mM}$ ammonium acetate and (B) methanol. The gradient elution was performed with $5 \%$ eluent $\mathrm{A}$ from 0 to $4 \mathrm{~min}$ and $80 \%$ eluent $\mathrm{A}$ from 4 to $4.5 \mathrm{~min}$.

Of the compounds in the cocktail substrate (i.e., melatonin, coumarin, omeprazole, dextromethorphan, tolbutamide and chlozoxazone), only the metabolite of chlozoxazone was able to be detected in negative ion mode, while the remaining metabolites were detected using the positive ion electrospray mode. The data were collected on a Micromass oa-Q-Tof (Waters) equipped with an electrospray ionization source. Basic parameter conditions included capillary, sample cone, and extraction cone voltages of 3500,30 , and $2 \mathrm{~V}$, respectively; desolvation and nebulization $\mathrm{N}_{2}$ flows of 450 and $45 \mathrm{l} / \mathrm{h}$, respectively; and desolvation and source temperatures of 350 and $120^{\circ} \mathrm{C}$, respectively. The mass spectrometer and UPLC systems were operated using Micromass MassLynx4.1 software. Chromatographic traces of protonated metabolites of CYP-specific substrates were extracted from total ion chromatograms. The masses of metabolites extracted at various retention times are shown in Table II.

Network of influence on metabolic enzymes of $G$. uralensis. In recent years, numerous studies (25-27) have shown that the different bioactive substances found in G. uralensis are metabolized by different CYP450 isoforms; at the same time, they are able to inhibit or activate the various isoforms of CYP450 to different degrees. In order to obtain a comprehensive understanding of the influence of $G$. uralensis on metabolic enzymes, a bioinformatics network of the influence of $G$. uralensis on metabolic enzymes through the PubMed literature database was created using Cytoscape software (28). The 47 documents selected through keyword retrieval from the PubMed database were imported to the Agilent Literature Search module of the Cytoscape software. A network figure was automatically generated by Cytoscape, and nodes and edges of the network were modified manually according to detailed information from the study abstracts. From this, a new network of the influence 
of G. uralensis on metabolic enzymes was produced, and a macroscopic overview of the effect of G. uralensis on metabolic enzymes was obtained.

Statistical analysis. Each experiment was performed twice. One-way analysis of variance (ANOVA) was used to analyze differences between samples, and $\mathrm{P}<0.05$ was considered to indicate statistically significant differences. The data for activity of the CYP450 enzyme were presented as the fold change compared with the control sample.

\section{Results}

Effect of components of G. uralensis on HepG2 cell viability. To determine whether GL, GA, L, IL, LG, LA and GC were cytotoxic, cell viability assays were performed. HepG2 cells were treated with serial dilutions $(0-50 \mu \mathrm{M})$ of GL, GA, L, IL, LG, LA or GC for $24 \mathrm{~h}$, and the cell survival rate was evaluated. Cell viability was not affected by any of the compounds at concentrations $<50 \mu \mathrm{M}$ when compared with the control cells (Fig. 1). Therefore, concentrations of 50 and $25 \mu \mathrm{M}$ were used for all experiments on HepG2 cells.

Expression of CYP1A2 mRNA following treatment with components of G. uralensis. The CYP450 superfamily of drug metabolism enzymes has a vital role in metabolizing most drugs. CYP1A2 accounts for $>10 \%$ of hepatic CYP enzymes and is known to be regulated by the aryhydrocarbon receptor (AhR). Additionally, CYP1A2 is involved in the metabolism of numerous steroid hormones and procarcinogens. The present study assessed whether GL, GA, L, IL, L, LA and GC modulate CYP1A2 expression. HepG2 cells were treated with the above-mentioned compounds at 25 and $50 \mu \mathrm{M}$, respectively, for $24 \mathrm{~h}$, and CYP1A2 mRNA levels were analyzed using qPCR. No significant difference was observed following treatment with LA (Fig. 2). By contrast, treatment with $25 \mu \mathrm{M}$ GL, L or IL induced a significant increase in CYP1A2 expression compared with the controls. However, when the cells were treated with $50 \mu \mathrm{M}$ GL, L or IL, CYP1A2 mRNA levels were significantly down-regulated. GA inhibited CYP1A2 expression in a dose-dependent manner; however, the inhibitory effects of LG and GC were not dose-dependent. The results indicated that the latter compounds may interact with each other to modulate CYP1A2 expression.

Expression of CYP2D6 $m R N A$ following treatment with G. uralensis. The effects of the components of G. uralensis on CYP2D6 expression were investigated. CYP2D6 is known for being polymorphic, and $30 \%$ of CYP-metabolized drugs are metabolized by CYP2D6. The results showed that GL, GA, L, IL, LG and LA significantly inhibited the expression of CYP2D6 transcripts (Fig. 3). However, following treatment with GC, a mixture of all the compounds, the expression of CYP2D6 was increased. This may be due to interactions between the different components of the mixture.

Expression of CYP2E1 $m R N A$ following treatment with G. uralensis-derived compounds. Expression of CYP2E1 mRNA did not differ significantly following treatment with GL. However, exposure to $25 \mu \mathrm{M} \mathrm{GA}, \mathrm{L}$ or GC inhibited

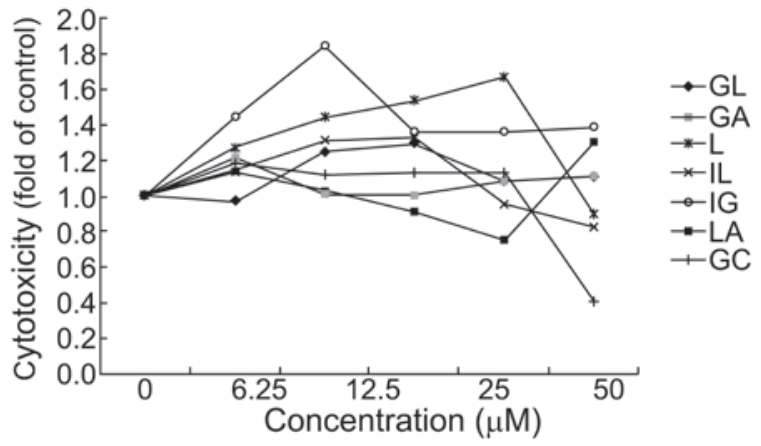

Figure 1. Effects of GL, GA, L, IL, LG, LA and GC on HepG2 cell viability. HepG2 cell viability was tested by MTT assay following incubation with 0-50 $\mu \mathrm{M}$ GL, GA, L, IL, LG, LA and GC, respectively, for $24 \mathrm{~h}$. GA, glycyrrhetinic acid; GL, glycyrrhizic acid; L, liquiritigenin; IL, isoliquiritigenin; LG, liquiritin; LA, licochalcone A; GC, equimolar mixture of GA, GL, L, IL, LG and LA.

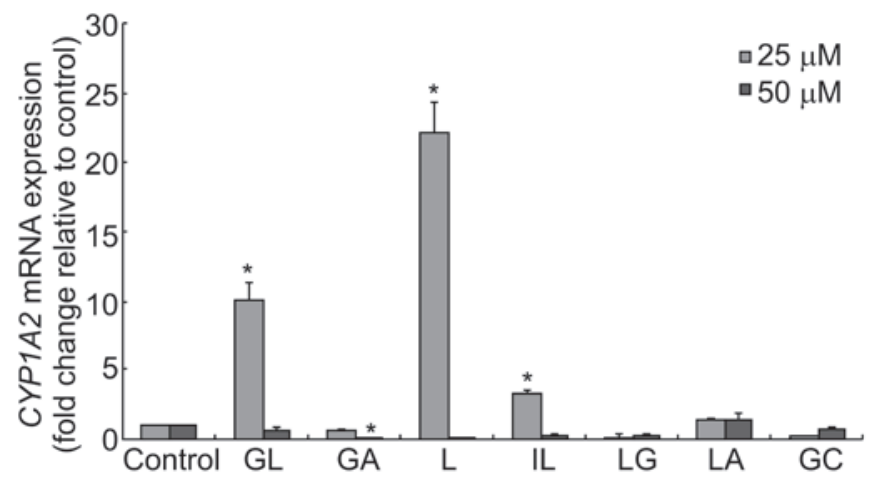

Figure 2. CYP1A2 mRNA levels following treatment with the various compounds. HepG2 cells were treated with 25 or $50 \mu \mathrm{M} \mathrm{GL}, \mathrm{GA}, \mathrm{L}$, IL, LG, LA or GC for $24 \mathrm{~h}$. The relative expression of CYP1A2 mRNA was assessed using qPCR. Fold change values were determined by normalizing to GADPH expression, and values were expressed as the fold change relative to the control. Data are presented as the mean \pm standard deviation ( $\mathrm{P}<0.05$ versus control). qPCR, quantitative polymerase chain reaction; CYP, cytochrome P; GA, glycyrrhetinic acid; GL, glycyrrhizic acid; L, liquiritigenin; IL, isoliquiritigenin; LG, liquiritin; LA, licochalcone A; GC, equimolar mixture of GA, GL, L, IL, LG and LA.

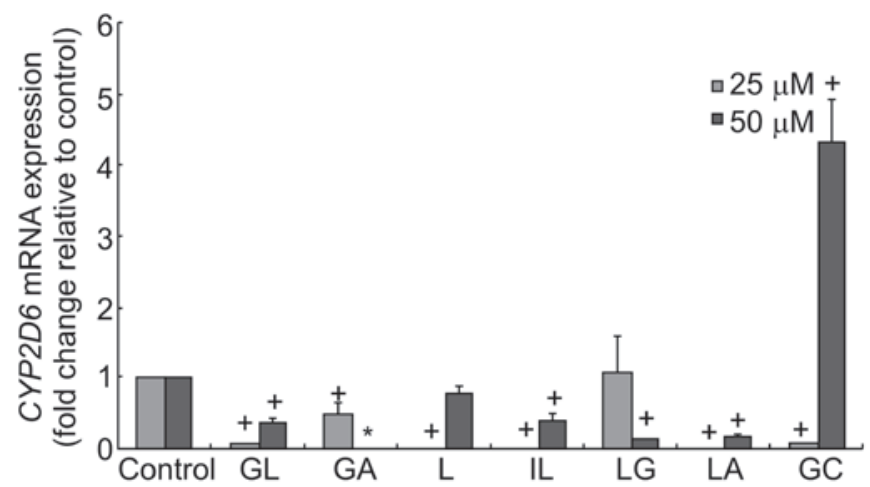

Figure 3. CYP2D6 mRNA levels following treatment with G. uralensis-derived compounds. HepG2 cells were treated with 25 or $50 \mu \mathrm{M}$ of GL, GA, L, IL, LG, LA or GC for $24 \mathrm{~h}$. The relative expression of CYP2D6 mRNA was assessed using qPCR. Fold change values were determined by normalizing to GADPH expression, and values were expressed as the fold change relative to the control. Data are presented as the mean \pm standard deviation $\left({ }^{*} \mathrm{P}<0.05\right.$ versus control). CYP, cytochrome P; GA, glycyrrhetinic acid; GL, glycyrrhizic acid; L, liquiritigenin; IL, isoliquiritigenin; LG, liquiritin; LA, licochalcone A; GC, equimolar mixture of GA, GL, L, IL, LG and LA. 


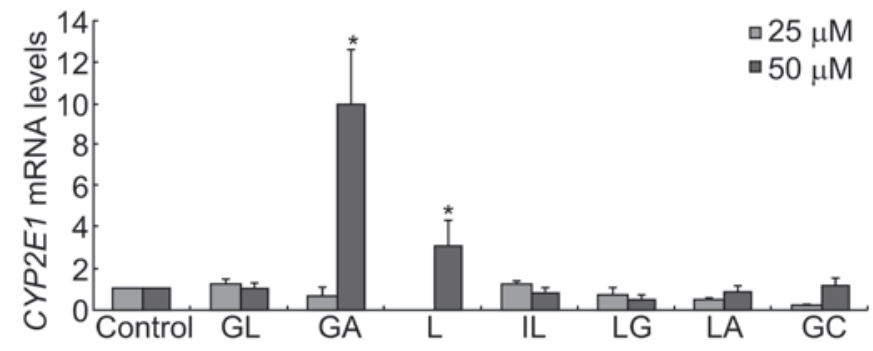

Figure 4. CYP2E1 mRNA levels after treatment with the G. uralensisderived compounds. HepG2 cells were treated with 25 or $50 \mu \mathrm{M}$ GL, GA, $\mathrm{L}$, IL, LG, LA, or GC for $24 \mathrm{~h}$. The relative expression of CYP2E1 mRNA was determined by real-time PCR. Fold-change values were determined by normalizing to GAPDH expression, and values were expressed as the fold change relative to the control. Data are presented as the mean \pm standard deviation $\left({ }^{*} \mathrm{P}<0.05\right.$ versus control).

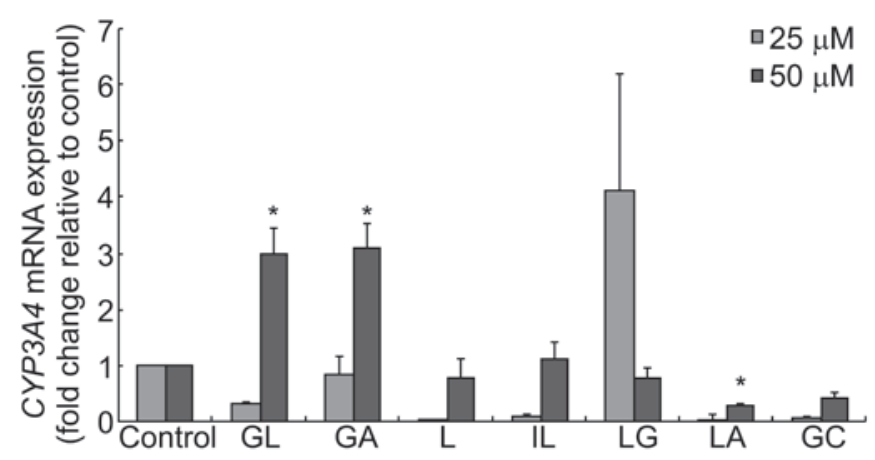

Figure 5. CYP3A4 mRNA levels following treatment with compounds derived from G. uralensis. HepG2 cells were treated with 25 or $50 \mu \mathrm{M} \mathrm{GL}$, GA, L, IL, LG, LA or GC for $24 \mathrm{~h}$. The relative expression of CYP3A4 mRNA was determined by qPCR. Fold change values were determined by normalizing to GAPDH expression, and values were expressed as the fold change relative to the control. Data are presented as the mean \pm standard deviation $($ $\mathrm{P}<0.05$ versus control). qPCR, quantitative polymerase chain reaction; CYP, cytochrome P; GA, glycyrrhetinic acid; GL, glycyrrhizic acid; L, liquiritigenin; IL, isoliquiritigenin; LG, liquiritin; LA, licochalcone A; GC, equimolar mixture of GA, GL, L, IL, LG and LA.

the expression of CYP2E1 transcripts, while $50 \mu \mathrm{M} \mathrm{GA}, \mathrm{L}$ or GC significantly increased the expression of CYP2E1.

Previous studies showed that the hepatoprotective effect of these compounds may be due to their ability to block the bioactivation of carbon tetrachloride by inhibiting P450 2E1 activity and expression (29-31). The present study showed that GL did not inhibit CYP2E1 expression (Fig. 4). However, IL and LG inhibited the expression of CYP2E1 transcripts in a dose-dependent manner. GA and L inhibited the expression of CYP2E1 transcripts when used at a low concentration $(25 \mu \mathrm{M})$, which is consistent with the study by Jeong et al (32), which showed that GA was able to inhibit the expression and activity of P450 2E1 and had protective effects against carbon tetrachloride-induced hepatotoxicity; however, they significantly increased $C Y P 2 E 1$ expression at a high concentration $(50 \mu \mathrm{M})$. However, when HepG2 cells were treated with a mixture of the six bioactive constituents of G. uralensis $(25 \mu \mathrm{M})$, the expression of CYP2E1 was slightly inhibited.

Expression of CYP3A4 mRNA following treatment with G. uralensis-derived compounds. CYP3A4, the major isoform
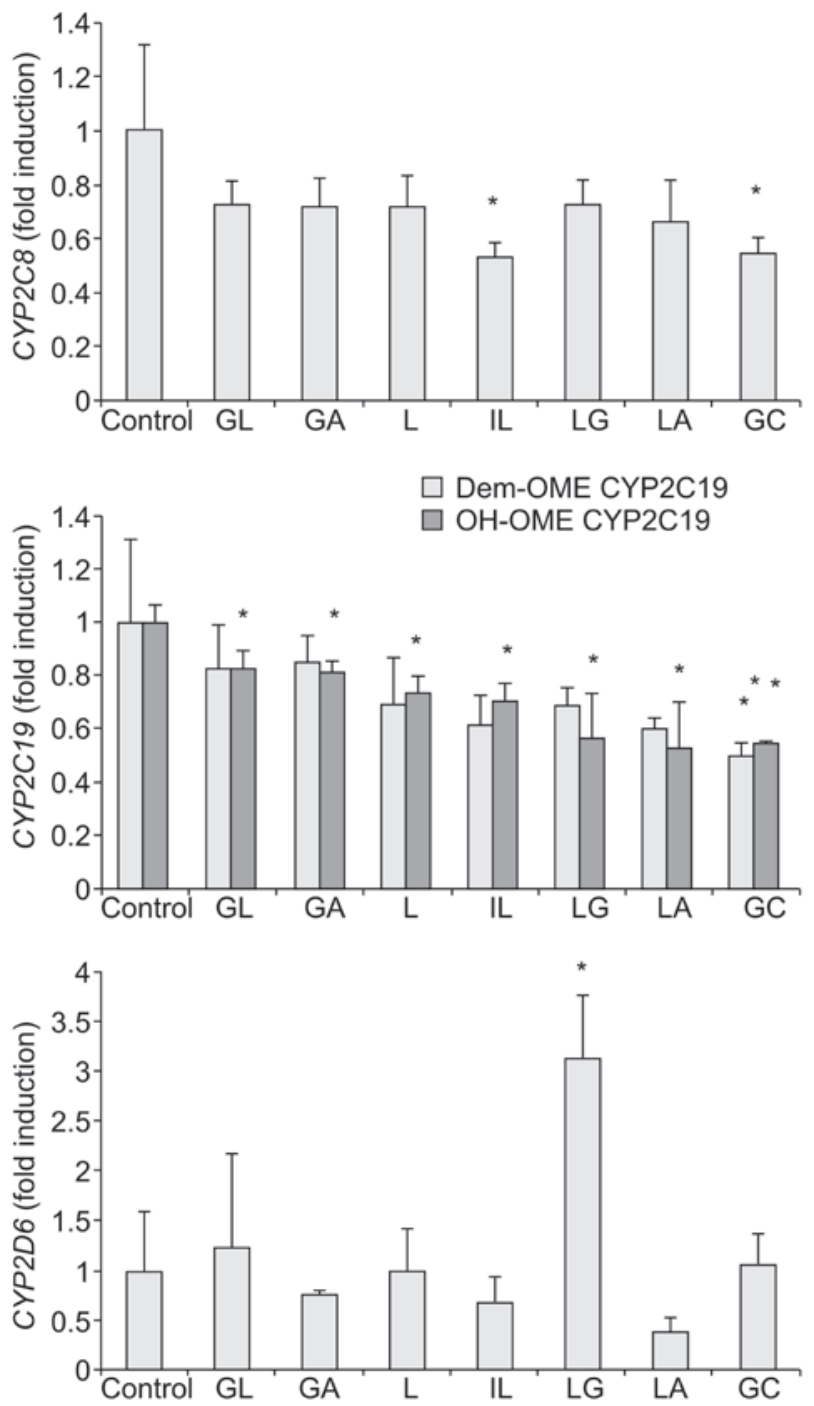

Figure 6. Effects of $25 \mu \mathrm{M}$ GL, GA, L, IL , LG, LA, and GC on the activities of CYP450 enzymes. Enzyme activities were measured by treatment of the cultures with the substrate cocktail and subsequent determination of the metabolites by LC-MS/MS. Results are shown as the mean \pm standard deviation $\left({ }^{*} \mathrm{P}<0.05\right.$ versus control). Analytical measurements were performed in duplicate. LC-MS/MS, liquid chromatography-tandem mass spectrometry; CYP, cytochrome P; GA, glycyrrhetinic acid; GL, glycyrrhizic acid; L, liquiritigenin; IL, isoliquiritigenin; LG, liquiritin; LA, licochalcone A; GC, equimolar mixture of GA, GL, L, IL, LG and LA.

of the CYP3A subfamily in humans, is the most important drug-metabolizing enzyme. As shown in Fig. 5, GL and GA increased the expression of CYP3A4 at a concentration of $50 \mu \mathrm{M}$, and GL, GA, L, IL, LG LA and GC modulated the expression of CYP3A4 in a dose-dependent manner.

CYP450 activity in HLMs following treatment with compounds derived from $G$. uralensis. Based on preliminary experiments in HLMs (33), appropriate probe substrates were selected as follows: Melatonin (CYP1A2), coumarin (CYP2A6), amodiaquine (CYP2C8), tolbutamide (CYP2C9), omeprazole (CYP1C19 and CYP3A4), dextromethorphan (CYP2D6) and chlozoxazone (CYP2E1). Cocktail activity assays were then performed in cultures of HLMs to assess all seven P450 enzymes simultaneously following treatment with the bioactive compounds from G. uralensis for $20 \mathrm{~min}$. Compared with the 


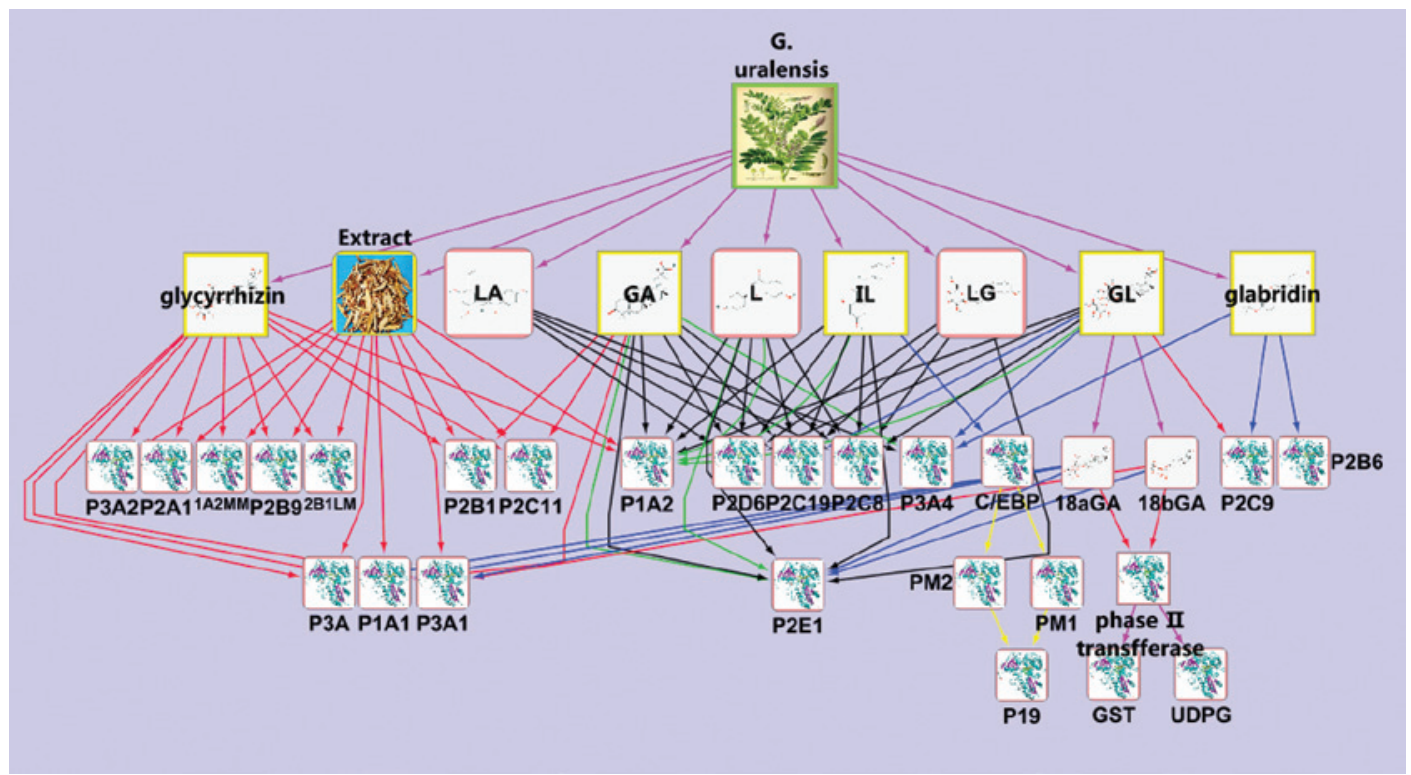

Figure 7. Network of influence of bioactive compounds derived from G. uralensis on metabolic enzymes (red arrow: activating effect according to the literature; blue arrow, inhibition according to the literature; green arrow, activating effect shown in the present study; black arrow, inhibition shown in present study).

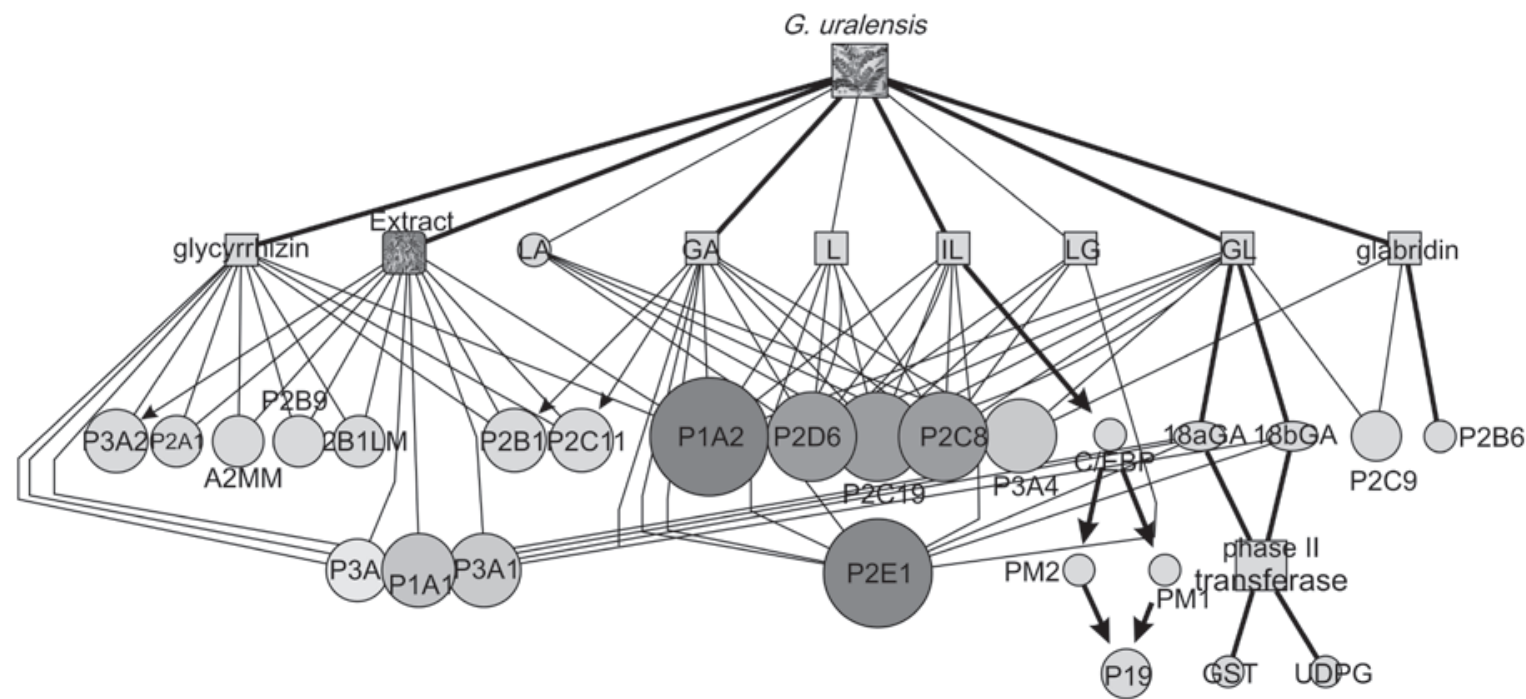

Figure 8. Analysis of the above network. Node sizes were determined by the degree of a node.

control sample, IL and GC strongly inhibited the activity of CYP2C8 and all the compounds derived from G. uralensis as well as their mixture (GC) significantly inhibited the activity of CYP2C19 (Fig. 6). LG showed the highest activity, inducing a 3 -fold increase in the expression of the CYP2D6 enzyme.

When detecting the metabolites of certain probes following treatment of HLMs with the bioactive compounds from G. uralensis, the activity of CYP1A2, -2A6, -2C9, -2E1, and $-3 \mathrm{~A} 4$ were not able to be determined.

Network of influence of bioactive compounds from G. uralensis on metabolic enzymes. The overview of the network of influences of G. uralensis-derived compounds on metabolic enzymes is shown in Fig. 7; this network was created through integration of data found in the literature and the results of the present study. The network was then analysed using the network analysis module in Cytoscape (Fig. 8). This analysis provided additional evidence that the compounds GL, GA, L, and IL, derived from $G$. uralensis, had effects on metabolic enzymes, particularly CYP450 family enzymes, and CYP1A, $-2 \mathrm{D},-2 \mathrm{C}$, $-2 \mathrm{E},-3 \mathrm{~A}$, and $-2 \mathrm{~A}$ were most susceptible to regulation by the bioactive components in G. uralensis.

\section{Discussion}

The present study aimed to reveal the correlation between G. uralensis and CYP450 enzymes. Consequently, the effect of the major bioactive constituents of G. uralensis on the expression of CYP isoforms in HepG2 cells was assessed using qPCR and the effect of the compounds on the activity of certain CYP450-associated enzymes in human liver microsomes was assessed using the cocktail assay. 
CYPP450s constitute a superfamily of membrane-bound heme proteins that catalyze the oxidative metabolism of a variety of endogenous compounds and environmental pollutants. Seven human isoforms of CYP are responsible for metabolizing $>90 \%$ of drugs currently used in the clinic (8). Alternatively, certain drugs are able to modify the expression and activity of CYP450 enzymes, thereby changing drug efficacy and pharmacokinetics. Therefore, it is highly important to study the significance of CYP enzymes in the regulation of drug efficacy.

The current medicinal applications of G. uralensis include regulating drug properties, improving spleen function and blood circulation as well as reducing cough. Regulating the activity of other drugs is the unique pharmacological trait of G. uralensis and is documented as an important concept in TCM. It is the most widely used medicinal plant in TCM that is co-administered with other medicines mainly due to its function of regulating the activity of drugs. With the wide use of G. uralensis, it is becoming increasingly important to be cautious about the interactions between $G$. uralensis and other drugs to avoid reduction in efficacy and increased side effects of co-administered drugs. However, studies in this field are rare. Previous studies (34) have shown that G. uralensis or its components were able to affect the activity of certain isoforms of CYP450 and pharmacokinetics of drugs. However, the specific isoforms of CYP450 involved and the effects of specific components of G. uralensis remain to be elucidated.

Consistent with the results of the present study, it has been reported that aqueous extracts of $G$.uralensis are able to activate the nuclear receptor PXR, and activated PXR is known to induce CYP3A expression $(22,35)$. Additionally, it has been demonstrated that GA significantly inhibits the activity of CYP3A4 in HLMs $(36,37)$. The signalling mechanisms mediating these processes may be diverse. Furthermore, CYP3A4 is regulated by several nuclear receptors, including the constitutive androstane receptor (CAR), PXR and the glucocorticoid receptor (GR). It has been reported that $\mathrm{L}$ is able to inhibit CYP3A4 in vitro (38), which is consistent with the results of the present study.

LG and LA are bioactive constituents of G. uralensis. These two compounds have various biological activities, including antioxidative, anti-inflammatory and anticarcinogenic activities. However, it remains unknown how these two compounds modulate the expression of CYP450 enzymes. The present study is, to the best of our knowledge, the first study to report the effects of LG and LA on the expression of CYP in HepG2 cells. It was demonstrated that LG inhibited the expression of $C Y P 1 A 2$ and $C Y P 2 E 1$ and induced the expression of $C Y P 2 D 6$ and $C Y P 3 A 4$ HepG2 cells at a concentration of $25 \mu \mathrm{M}$. Moreover, these compounds inhibited the expression of CYP2D6 and CYP3A4 at a higher concentration $(50 \mu \mathrm{M})$. The results showed that LA effectively inhibited the expression of $C Y P 2 D 6, C Y P 2 E 1$, and $C Y P 3 A 4$, but did not affect $C Y P 1 A 2$ expression.

The results demonstrating the effects of these compounds on CYP2D6 activity were similar to the qPCR data showing the effects of the compounds on $C Y P 2 D 6$ gene expression. Moreover, the cocktail assay allowed for determination of the activity of CYP2C8 and CYP2C19, which are expressed at very low levels in HepG2 cells. Of note, the data for the two latter enzymes were highly distinctive from the results of the gene expression analysis as well as the gene expression and cocktail results in the present study, which may be due to the system used in these experiments and the concentrations of compounds used to treat the cells. Moreover, certain compounds may have induced or inhibited the expression of genes and affected the activity of proteins through diverse pathways.

Through the overview of the bioinformatics network of the influence of $G$. uralensis-derived compounds on metabolic enzymes, increasing evidence has shown that the compounds GA, IL, GL, and L, derived from G. uralensis, had effects on metabolic enzymes, particularly CYP450 family enzymes, and CYP1A, -2D, -2C, -2E, -3A and -2A were most susceptible to regulation by the bioactive components of $G$. uralensis. This showed the major targets among the CYP450 enzymes in regard to the modulation of drug functions and properties by $G$. uralensis.

Due to the complexity of the components of G. uralensis and pathophysiology, it is very difficult to fully elucidate the mechanisms of the modulation of the activity of drugs exclusively from the aspect of metabolic enzymes. Thus, the present study was incomplete, and a metabolomics study could be conducted to explain the mechanism from the aspect of endogenous metabolites.

In conclusion, the present study has demonstrated that the bioactive constituents of $G$. uralensis differentially modulated the expression of CYP enzymes. Moreover, a combination of gene expression studies using qPCR and activity assays for CYP450 in HLMs using cocktail probes and UPLC-MS/MS analysis allowed for correlation between Chinese Herbal Medicine and CYP450 enzymes. The findings of the present study are useful and may aid physicians to avoid risks and side effects caused by interactions between $G$. uralensis extracts and conventional drugs metabolized by these enzymes. The present study also sheds light on the mechanism by which G. uralensis modulates the properties of other drugs in the context of metabolism by CYP450 expression.

\section{Acknowledgements}

The authors thank Professor Gengfu Chen, School of Basic Courses, Guangdong Pharmaceutical University, for his guidance in the experiments. The present study was supported by the National Natural Science Foundation of China (no. 30801543).

\section{References}

1. Nelson DR, Koymans L, Kamataki T, et al: P450 superfamily: update on new sequences, gene mapping, accession numbers and nomenclature. Pharmacogenetics 6: 1-42, 1996.

2. Hodgson E and Rose RL: The importance of cytochrome P450 2B6 in the human metabolism of environmental chemicals. Pharmacol Ther 113: 420-428, 2007.

3. Hodgson E and Rose RL: Metabolic interactions of agrochemicals in humans. Pest Manag Sci 64: 617-621, 2008.

4. Shimazu S, Inui $\mathrm{H}$ and Ohkawa $\mathrm{H}$ : Phytomonitoring and phytoremediation of agrochemical and related compounds based on recombinant cytochrome P450s and aryl hydrocarbon receptors (AhRs). J Agric Food Chem 59: 2870-2875, 2011.

5. Naiman K, Frei E and Stiborova M: Identification of rat cytochromes P450 metabolizing N-(2-methoxyphenyl)hydroxylamine, a human metabolite of the environmental pollutants and carcinogens o-anisidine and o-nitroanisole. Neuro Endocrinol Lett 31 (Suppl 2): 36-45, 2010.

6. Uppstad H, Øvrebø S, Haugen A and Mollerup S: Importance of CYP1A1 and CYP2B1 in bioactivation of benzo[a]pyrene in human lung cell lines. Toxicol Lett 192: 221-228, 2010. 
7. Sanada N, Gotoh Y, Shimazawa R, Klinge CM and Kizu R Repression of activated aryl hydrocarbon receptor-induced transcriptional activation by $5 \alpha$-dihydrotestosterone in human prostate cancer LNCaP and human breast cancer T47D cells. J Pharmacol Sci 109: 380-387, 2009.

8. Park BK: Cytochrome P450 enzymes in the heart. Lancet 355: 945-946, 2000

9. Cui X, Thomas A, Han Y, et al: Quantitative PCR assay for cytochromes $\mathrm{P} 450$ 2B and $3 \mathrm{~A}$ induction in rat precision-cut liver slices: correlation study with induction in vivo. J Pharmacol Toxicol Methods 52: 234-243, 2005.

10. Martignoni M, de Kanter R, Grossi P, Saturno G, Barbaria E and Monshouwer M: An in vivo and in vitro comparison of CYP gene induction in mice using liver slices and quantitative RT-PCR. Toxicol In Vitro 20: 125-131, 2006.

11. Deng Y, Bi HC, Zhao LZ, et al: Induction of cytochrome P450 3A by the Ginkgo biloba extract and bilobalides in human and rat primary hepatocytes. Drug Metab Lett 2: 60-66, 2008.

12. Lobo ED, Bergstrom RF, Reddy S, et al: In vitro and in vivo evaluations of cytochrome P450 1A2 interaction with duloxetine. Clin Pharmacokinet 47: 191-202, 2008.

13. Sparfel L, Payen L, Gilot D, et al: Pregnane X receptor-dependent and -independent effects of 2-acetylaminofluorene on cytochrome P450 3A23 expression and liver cell proliferation. Biochem Biophys Res Commun 300: 278-284, 2003.

14. Volotinen M, Mäenpää J, Kankuri E, et al: Expression of cytochrome P450 (CYP) enzymes in human nonpigmented ciliary epithelial cells: induction of CYP1B1 expression by TCDD. Invest Ophthalmol Vis Sci 50: 3099-3105, 2009.

15. Westerink WM and Schoonen WG: Cytochrome P450 enzyme levels in HepG2 cells and cryopreserved primary human hepatocytes and their induction in HepG2 cells. Toxicol In Vitro 21: 1581-1591, 2007.

16. Saxena A, Tripathi KP, Roy S, Khan F and Sharma A: Pharmacovigilance: effects of herbal components on human drugs interactions involving cytochrome P450. Bioinformation 3: 198-204, 2008.

17. Kennedy DA and Seely D: Clinically based evidence of drug-herb interactions: a systematic review. Expert Opin Drug Saf 9: 79-124, 2010.

18. Yang XX, Hu ZP, Duan W, Zhu YZ and Zhou SF: Drug-herb interactions: eliminating toxicity with hard drug design. Curr Pharm Des 12: 4649-4664, 2006

19. Borrelli F and Izzo AA: Herb-drug interactions with St John's Wort (Hypericum perforatum): an update on clinical observations. AAPS J 11: 710-727, 2009.

20. Zhang Q and Ye M: Chemical analysis of the Chinese herbal medicine Gan-Cao (licorice). J Chromatogr A 1216: 1954-1969, 2009.

21. Asl MN and Hosseinzadeh H: Review of pharmacological effects of Glycyrrhiza sp. and its bioactive compounds. Phytother Res 22: 709-724, 2008.

22. Tang J, Song X, Zhu M and Zhang J: Study on the pharmacokinetics drug-drug interaction potential of Glycyrrhiza uralensis, a traditional Chinese medicine, with lidocaine in rats. Phytother Res 23: 603-607, 2009.
23. Ma T, Huang C, Zong G, Zha D, Meng X, Li J and Tang W: Hepatoprotective effects of geniposide in a rat model of nonalcoholic steatohepatitis. J Pharm Pharmacol 63: 587-593, 2011.

24. Livak KJ and Schmittgen TD: Analysis of relative gene expression data using real-time quantitative PCR and the $2^{(-\Delta \Delta \mathrm{C}(\mathrm{T}))}$ Method. Methods 25: 402-408, 2001.

25. Zhao K, Ding M, Cao H and Cao ZX: In-vitro metabolism of glycyrrhetinic acid by human and rat liver microsomes and its interactions with six CYP substrates. J Pharm Pharmacol 64: 1445-1451, 2012.

26. Yu K, Chen F and Li C: Absorption, disposition, and pharmacokinetics of saponins from Chinese medicinal herbs: what do we know and what do we need to know more? Curr Drug Metab 13: 577-98, 2012

27. Liu L, Xiao J, Peng ZH and Chen Y: In vitro metabolism of glycyrrhetic acid by human cytochrome P450. Yao Xue Xue Bao 46: 81-87, 2011.

28. Smoot M, Ono K, Ruscheinski J, Wang PL and Ideker T: Cytoscape 2.8: new features for data integration and network visualization. Bioinformatics 27: 431-432, 2011

29. Fuhr U: Induction of drug metabolizing enzymes: pharmacokinetic and toxicological consequence in humans. Clin Pharmacokinet 38: 493-504, 2000.

30. Quan J, Yin X and Xu H: Boschniakia rossica prevents the carbon tetrachloride-induced hepatoxicity in rat. Exp Toxicol Pathol 63: 53-59, 2011.

31. Waluga $\mathrm{M}$ and Hartleb $\mathrm{M}$ : Alcoholic liver disease. Wiad Lek 56: 61-70, 2003.

32. Jeong HG, You HJ, Park SJ, Moon AR, Chung YC, Kang SK, and Chun HK: Hepatoprotective effects of 18 beta-glycyrrhetinic acid on carbon tetrachloride-induced liver injury: inhibition of cytochrome P450 2E1 expression. Pharmacol Res 46: 221-227, 2002.

33. Alden PG, Plumb RS, Jones MD, Rainville PD and Shave D: A rapid ultra-performance liquid chromatography/tandem mass spectrometric methodology for the in vitro analysis of Pooled and Cocktail cytochrome P450 assays. Rapid Commun Mass Spectrom 24: 147-154, 2010

34. Paolini M, Barillari J, Broccoli M, Pozzetti L, Perocco P and Cantelli-Forti G: Effect of liquorice and glycyrrhizin on rat liver carcinogen metabolizing enzymes. Cancer Lett 145: 35-42, 1999.

35. Mu Y, Zhang J, Zhang S, et al: Traditional Chinese medicines Wu Wei $\mathrm{Zi}$ (Schisandra chinensis Baill) and Gan Cao (Glycyrrhiza uralensis Fisch) activate pregnane $\mathrm{x}$ receptor and increase warfarin clearance in rats. J Pharmacol Exp Ther 316: 1369-1377, 2006.

36. Li HY, Xu W, Su J, Zhang X, Hu LW and Zhang WD: In vitro and in vivo inhibitory effects of glycyrrhetinic acid on cytochrome P450 3A activity. Pharmacology 86: 287-292, 2010.

37. Liu L, Xiao J, Peng ZH and Chen Y: In vitro metabolism of glycyrrhetic acid by human cytochrome P450. Yao Xue Xue Bao 46: 81-87, 2011.

38. Tsukamoto S, Aburatani M, Yoshida T, Yamashita Y, El-Beih AA and Ohta T: CYP3A4 inhibitors isolated from Licorice. Biol Pharm Bull 28: 2000-2002, 2005. 